\title{
Magnetic Flux Transport in Protostellar Accretion Disks
}

\section{John Contopoulos and Arieh Königl}

Dept. of Astronomy \& Astrophysics, Univ. of Chicago, 5640 S. Ellis Ave., Chicago, IL 60637, U.S.A.

\begin{abstract}
Centrifugally driven winds from the surfaces of magnetized accretion disks are a leading candidate for the origin of bipolar outflows and have also been recognized as an attractive mechanism for removing the angular momentum of the accreted matter. The origin of the open magnetic field lines that thread the disk in this scenario is, however, still uncertain. One possibility is that the field lines are transported through the disk, but previous studies have shown that this process is inefficient in disks with turbulent viscosity and diffusivity. Here we examine whether the efficiency can be increased if angular momentum is transported from the disk surfaces by large-scale magnetic fields instead of radially by viscous stresses. In this picture, the removal of angular momentum is associated with the establishment of a global poloidal electric current driven by the radial EMF in the disc, and it does not necessarily need to involve super-Alfvénic outflows. We address this problem in the context of protostellar systems and present representative solutions of the time evolution of a resistive disk that is initially threaded by a uniform vertical field anchored at a large distance from its surfaces. We assume that the angular momentum transport in the disk is controlled by the large-scale magnetic field and take into account the influence of the field on the disk structure.
\end{abstract}

